\title{
CONTENTS OF VOLUMES $1-13$
}

\author{
Alphabetized by Authors' Names
}

Aarsleff, Hans

The Eighteenth Century, Including Leibniz . . . . . . . . . . . 13383

Abboud, Peter F.

Spoken Arabic . . . . . . . . . . . . . . . . . . . . 6439

Abernathy, Robert

Mathematical Linguistics . . . . . . . . . . . . . . . . . 1113

Abramson, Arthur S.

Phonetics: An Overview . . . . . . . . . . . . . . . . . 122187

Akira, $\overline{\text { Ota }}$

The Study of English in Japan . . . . . . . . . . . . . 2645

Akiyasu, Tỏo

Postwar Studies of the Chinese Language . . . . . . . . . . . . 2633

Alexandre, Pierre

Multilingualism . . . . . . . . . . . . . . . . . . . 7654

Alisjahbana, S.T.

see Wurm, Stephen A. . . . . . . . . . . . . . . . . . . 81025

Allen, Harold B.

English as a Second Language . . . . . . . . . . . . . . . 10295

Althaus, Hans Peter

Yiddish . . . . . . . . . . . . . . . . . . . . . . . 91345

Altoma, Salih J.

Language Education in Arab Countries and the Role of the Academies . . 6690 Anisfeld, Moshe

Social Perception of Speech . . . . . . . . . . . . . . . . 121429

Ansre, Gilbert

Language Standardisation in Sub-Saharan Africa . . . . . . . . . 7680

Anttila, Raimo

Internal Reconstruction and Finno-Ugric (Finnish) . . . . . . . . 11317

Applegate, Joseph R.

The Berber Languages . . . . . . . . . . . . . . . . . . 6586

Austerlitz, Robert

History of Phonetics: A Bibliography . . . . . . . . . . . . . 131179

Avis, Walter S.

The English Language in Canada . . . . . . . . . . . . . . . 1040 Bahl, Kali Charan

Panjabi 
Bar-Hillel, Yehoshua, Jonathan Malino, Avishai Margalit

On Logic and Theoretical Linguistics . . . . . . . . . . . . . 1237

Barthel, Thomas S.

Linguistics of Non-Ibero-American Linguistics: Writing Systems . . . . 4275

Pre-contact Writing in Oceania . . . . . . . . . . . . . . . 81165

Bellugi, Ursula

Some Aspects of Language Acquisition . . . . . . . . . . . . 121136

Bender, Ernest

Middle Indo-Aryan . . . . . . . . . . . . . . . . . . . . . . 546

Bender, Byron W.

Micronesian Languages . . . . . . . . . . . . . . . . . . 8426

Bendor-Samuel, John T.

Niger-Congo, Gur . . . . . . . . . . . . . . . . . . . 7141

Benveniste, Emile

Old Iranian . . . . . . . . . . . . . . . . . . . . . . 69

Bernstein, Basil B.

Social, Class, Language, and Socialization . . . . . . . . . . . . 121545

Berry, Jack

Pidgins and Creoles in Africa . . . . . . . . . . . . . . . . . 7510

Bever, T.G.

The Interaction of Perception and Linguistic Structures: A Preliminary

Investigation of Neo-Functionalism . . . . . . . . . . . . . . 121159

Bierwisch, Manfred

Generative Grammar and European Linguistics . . . . . . . . . . 9313

Biggs, Bruce

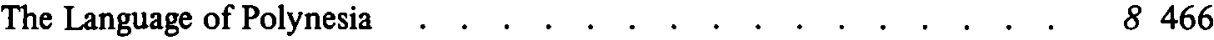

Biligiri, H.S.

Kannada . . . . . . . . . . . . . . . . . . . . . . 5394

Blanc, Haim

Semitic . . . . . . . . . . . . . . . . . . . . . . 1374

Arabic . . . . . . . . . . . . . . . . . . . . . . . 7501

Linguistics among the Arabs . . . . . . . . . . . . . . . . 131265

Blanch, Juan M. Lope

Hispanic Dialectology . . . . . . . . . . . . . . . . . . 4106

Blaylock, Curtis

General and Serial Publications Relevant to the Area and the Fields . . . 91819

Blumenthal, Arthur L.

An Historical View of Psycholinguistics . . . . . . . . . . . . 121105

Bodman, Nicholas Cleaveland

Historical Linguistics . . . . . . . . . . . . . . . . . . 23

Bowen, J. Donald

Hispanic Languages and Influence in Oceania . . . . . . . . . . 8938

Brekle, Herbert E.

The Seventeenth Century . . . . . . . . . . . . . . 13277

Bright, William

North American Indian Language Contact . . . . . . . . . . . 10713 
Bronowski, J.

Language in a Biological Frame . . . . . . . . . . . . . . . 122539

Bursill-Hall, G. L.

The Middle Ages . . . . . . . . . . . . . . . . . . . . 13179

Burrow, Thomas

Sanskrit . . . . . . . . . . . . . . . . . . . . 53

Câmara Jr., Joaquim Mattoso

Brazilian Linguistics . . . . . . . . . . . . . . . . . . . 4229

Capell, A.

The Austronesian Languages of Australian New Guinea . . . . . . . 8240

History of Research in Australian and Tasmanian Languages . . . . . 8661

Cassidy, Frederic G.

Dialect Studies, Regional and Social . . . . . . . . . . . . . 1075

Castañeda, Leonardo Manrique

Computational Linguistics . . . . . . . . . . . . . . . . . 4543

Catalán, Diego

Ibero-Romance . . . . . . . . . . . . . . . . . . . . 9927

Catford, J. C.

Phonetic Fieldwork . . . . . . . . . . . . . . . . . . . 122489

Cazacu, Boris

Romanian . . . . . . . . . . . . . . . . . . . . . . 91145

Chadwick, John

Linear B . . . . . . . . . . . . . . . . . . . . . . 11537

Chafe, Wallace $L$.

Siouan, Iroquoian, and Caddoan . . . . . . . . . . . . . . . 101164

Chang, Kun

Descriptive Linguistics . . . . . . . . . . . . . . . . . . 259

National Languages . . . . . . . . . . . . . . . . . . . 2151

Chatman, Seymour and Samuel R. Levin

Linguistics and Literature . . . . . . . . . . . . . . . . . 10250

Chomsky, Noam

Topics in the Theory of Generative Grammar . . . . . . . . . . 31

Bibliography . . . . . . . . . . . . . . . . . . 358

Cicourel, Aaron V.

Ethnomethodology . . . . . . . . . . . . . . . . . . . 121563

Cisneros, Luis Jaime

Spanish . . . . . . . . . . . . . . . . . . . . . . . 4379

Cizikova, Ksenija, and Charles A. Ferguson

Bibliographical Review of Bengali Studies . . . . . . . . . . . 585

Clark, Herbert $\mathrm{H}$.

Semantics and Comprehension . . . . . . . . . . . . . . . 121291

Clark, T. W.

Nepali and Pahari . . . . . . . . . . . . . . . . . . . 5249

Clement, Dorothy and Benjamin N. Colby

Folk Narrative

12809 
XXXII

Cohen, L. Jonathan

Speech Acts . . . . . . . . . . . . . . . . . . . . . . . . . . . 12173

Colby, Benjamin N.

see Clement, Dorothy . . . . . . . . . . . . . . . . . 12809

Cole, Desmond T.

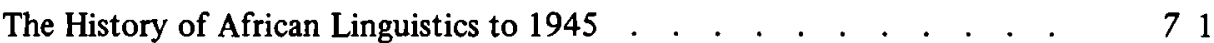

Connolly, M. J.

Synchronic Armenian . . . . . . . . . . . . . . . . . . 6160

Constantino, Ernesto

Tagalog and Other Major Languages of the Philippines . . . . . . . 8112

Contreras, Heles

Applied Linguistic Research . . . . . . . . . . . . . . . . 4534

Cornyn, William S.

Burma . . . . . . . . . . . . . . . . . . . . . 2777

Coseriu, Eugenio

General and Ibero-American Linguistics: General Perspectives . . . . . 45

Coseriu, Eugenio and Horst Geckeler

Linguistics and Semantics . . . . . . . . . . . . . . . . . 12103

Craddock, Jerry $\mathbf{R}$.

Spanish in North America . . . . . . . . . . . . . . . . . 10467

Crystal, David

Paralinguistics . . . . . . . . . . . . . . . . . . . . . 12265

Décsy, Gyula

Hungary . . . . . . . . . . . . . . . . . . . . 1523

DeFrancis, John

Language and Script Reform . . . . . . . . . . . . . . . . 2130

de Mauro, Tullio

Italian and Sardinian . . . . . . . . . . . . . . . . . . . 91128

De Silva, M. W. Sugathapala

Sinhalese . . . . . . . . . . . . . . . . . . . . . 5235

Devoto, Giacomo

Studies of Latin and Languages of Ancient Italy . . . . . . . . . 9817

Dil, Anwar S.

Linguistic Studies in Pakistan . . . . . . . . . . . . . . . . . 5679

Di Pietro, Robert J.

Bilingualism . . . . . . . . . . . . . . . . . . . . 4399

Doerfer, Gerhard

Irano-Altaistica: Turkish and Mongolian Languages of Persia and

Afghanistan . . . . . . . . . . . . . . . . . . . 6217

Dresden, Mark J.

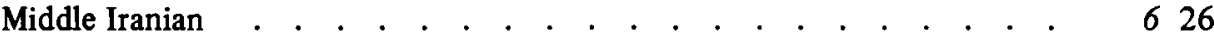

Dulong, Gaston

Histoire du français en Amérique du Nord . . . . . . . . . . . 10407

Dunstan, Elizabeth

Language Teaching . . . . . . . . . . . . . . . 7570 
Dyen, Isidore

The Austronesian Languages and Proto-Austronesian . . . . . . . . 85

The Austronesian Languages of Formosa . . . . . . . . . . . . 8168

The Chamic Languages . . . . . . . . . . . . . . . . . . 8200

Malagasy . . . . . . . . . . . . . . . . . . . . . 8211

Egerod, S $\phi$ ren

Dialectology . . . . . . . . . . . . . . . . . . . . . 291

Emeneau, Murray B.

The Non-Literary Dravidian Languages . . . . . . . . . . 5334

Engler, Rudolf

European Structuralism: Saussure . . . . . . . . . . . . . . 13829

Escobar, Alberto

Present State of Linguistics . . . . . . . . . . . . . . . . 4616

Fairbanks, Gordon H.

Comparative Indo-Aryan . . . . . . . . . . . . . . . . . 536

Ferguson, Charles A.

Regional Language Issues and Studies: An Overview . . . . . . . . 6665

Ferguson, Charles A.

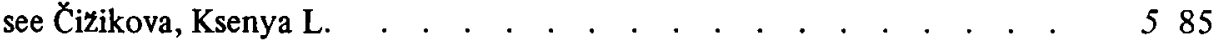

Fillenbaum, Samuel

Syntactic Factors in Memory . . . . . . . . . . . . . . . . 121235

Fischer, John L.

Style Contrasts in Pacific Languages . . . . . . . . . . . . . 81129

Fishman, Joshua A.

The Sociology of Language: An Interdisciplinary Social Science Approach

to Language in Society . . . . . . . . . . . . . . . . . . 121629

Foster, Philip J.

Problems of Literacy in Sub-Saharan Africa . . . . . . . . . . . 7587

Fought, John

see Hymes, Dell . . . . . . . . . . . . . . . . . . . . 13903

Fox, Joseph P.

see Taylor, Orlando L. . . . . . . . . . . . . . . . . . . 122595

Francis, W. Nelson

Approaches to Grammar . . . . . . . . . . . . . . . . . 10122

Freudenthal, $\mathrm{H}$.

Cosmic Language . . . . . . . . . . . . . . . . . . . . 121019

Fry, D. B.

Phonetics in the Twentieth Century . . . . . . . . . . . . . 122201

Fudge, Erik G.

Phonology and Phonetics . . . . . . . . . . . . . . . . . 9254

Garcia, Erica

Hispanic Phonology . . . . . . . . . . . . . . . . . . . 463

Garvin, Paul L.

Czechoslovakia . . . . . . . . . . . . . . . . . . . . 1499

Language Analysis . . . . . . . . . . . . . . . . . . . 122741

Specialty Trends in the Language Sciences . . . . . . . . . . . 122889 
XXXIV

Geckeler, Horst see Coseriu, Eugenio $\quad . \quad$. . . . . . . . . . . . . . . . . 12103

Gedney, William J.

Thailand and Laos . . . . . . . . . . . . . . . . 2782

Gelb, Ignace J.

Written Records and Decipherment . . . . . . . . . . . . . . 11253

Gipper, Helmut, und Peter Schmitter

Sprachwissenschaft und Sprachphilosophie im Zeitalter der Romantik . . 13481

Goddard, Ives

Philological Approaches to the Study of North American Indian

Languages: Documents and Documentation . . . . . . . . . . . 10727

Godel, Robert

Appendix l: F. de Saussure's Theory of Language . . . . . . . . . 3479

Diachronic Armenian . . . . . . . . . . . . . . . . . . 6139

Goląb, Zbigniew

Bulgaria . . . . . . . . . . . . . . . . . . . . . . . 1477

Golopenția-Eretescu, Sanda

Formalized Languages: Scientific . . . . . . . . . . . . . . 12537

Gomes de Matos, Francisco, and Leopoldo Wigdorsky

Foreign Language Teaching in Latin America . . . . . . . . . . 4464

Gonda, J.

The Influence of Indian Languages . . . . . . . . . . . 8955

Goodman, Morris

Languages in Contect . . . . . . . . . . . . . . . . . . 7664

Grace, George W.

Languages of the New Hebrides and Solomon Islands . . . . . . . . 8341

Greenberg, Joseph $\mathrm{H}$.

Language Universals . . . . . . . . . . . . . . . . . . . 361

Appendix on Word Association . . . . . . . . . . . . . . . 3111

Nilo-Saharan and Meroitic . . . . . . . . . . . . . . . . . 7421

The Indo-Pacific Hypothesis . . . . . . . . . . . . . . . . 8807

The Typological Method . . . . . . . . . . . . . . . . . 11149

Grimes, Joseph E.

Descriptive Linguistics . . . . . . . . . . . . . . . . . . 4302

Grognet, Allene Guss

United States Current Resources in Linguistics . . . . . . . . . . 101449

Grootaers, Willem A.

Dialectology . . . . . . . . . . . . . . . . . . . . . 2585

Gross, Maurice

New Formal Devices for Linguistics . . . . . . . . . . . . . 12985

Gudschinsky, Sarah C. $\dagger$

Linguistics and Literacy . . . . . . . . . . . . . . . . . 122039

Guiraud, Pierre

French . . . . . . . . . . . . . . . . . . . . . . . 91107

Rhetoric and Stylistics . . . . . . . . . . . . . . . . . 12943 
Guitarte, Guillermo L. , and Rafael Torres Quintero Linguistic Correctness and the Role of the Academies . . . . . . . . . 4562

Gumperz, John J.

Sociolinguistics in South Asia $\quad . \quad$. . . . . . . . . . . . . . 559

Gupta, Jyotirindra Das Official Language Problems and Politics in South Asia . . . . . . . 5578

Guthrie, Malcolm The Western Bantu Languages . . . . . . . . . . . . . . . 7357

Haas, Mary R.

Historical Linguistics and the Genetic Relationship of Languages . . . . 3113

1. Introductory Remarks . . . . . . . . . . . . . . . . 3113

2. Protolanguages and Problems of Reconstruction . . . . . . . 3.123

3. The Ranking of Protolanguages and Problems of Comparison at Deeper Levels . . . . . . . . . . . . . . . . 3138

4. Problems of Classification . . . . . . . . . . . . . . . 3145

5. Supplemental Methods . . . . . . . . . . . . . . . . 3152

American Indian Linguistic Prehistory . . . . . . . . . . . 10677

The Southeast . . . . . . . . . . . . . . . . . 101210

Haden, Ernest F.

French Dialect Geography in North America . . . . . . . . . . 10422

Hagedorn, William see Werner Oswald . . . . . . . . . . . . . . . . . . . . . . . . . . . . . 121477

Hale, Kenneth

Deep-Surface Canonical Disparities in Relation to Analysis and Change:

An Australian Example . . . . . . . . . . . . . . . . . . 11401

Hall, Jr. , Robert A.

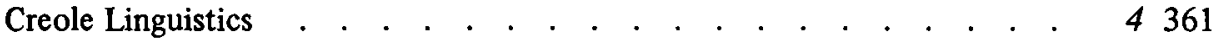

Halle, Morris

Phonemics . . . . . . . . . . . . . . . . . . . . . . 15

Hamp, Eric P.

Albanian . . . . . . . . . . . . . . . . . . . 91626

Harper, Kenneth E.

Machine Translation . . . . . . . . . . . . . . . . . . . . 133

Harris, Katherine S.

Physiological Aspects of Articulatory Behavior . . . . . . . . . . 122281

Hartmann, Peter, and Siegfried J. Schmidt

Theory and Philosophy of Language . . . . . . . . . . . . . 93

Hartman, Reinhard Rudolf Karl

The Organization of Linguistics in Western Europe . . . . . . . . 91795

Hattori, Shirô

Descriptive Linguistics in Japan . . . . . . . . . . . . . . . 2530

Japanese Dialects . . . . . . . . . . . . . . . . . . . . . . . . . 11369

Haudricourt, André G.

New Caledonia and the Loyalty Islands . . . . . . . . . . 8359 
Haugen, Einar

Bilingualism, Language Contact, and Immigrant Languages in the United

States: A Research Report 1956-1970 . . . . . . . . . . . . . 10505

Haugen, Einar and Thomas L. Markey

The Scandinavian Languages: Fifty Years of Linguistic Research . . . . 91461

Hays, David G.

Information Handling . . . . . . . . . . . . . . . . . . 122719

Hazai, G.

Turkish . . . . . . . . . . . . . . . . . . . . . . 6183

Linguistics and Language Issues in Turkey . . . . . . . . . . . 6746

Hécaen, Henry

Studies of Language Pathology . . . . . . . . . . . . . . . 9591

Heinz, John M.

Speech Acoustics . . . . . . . . . . . . . . . . . . . . 122241

Hendricks, William $\mathrm{O}$.

Linguistics and Folkloristics . . . . . . . . . . . . . . . . 12661

Hettiaratchi, D. E.

Linguistics in Ceylon, (I) . . . . . . . . . . . . . . . . . . . 5746

Hiroshi, Tsukishima

Historical Linguistics, Including Affiliations with Other Languages . . . 2503

Hockett, Charles F.

Language, Mathematics, and Linguistics . . . . . . . . . . . . 3155

0. Introduction . . . . . . . . . . . . . . . . . 3155

1. Mathematical Background . . . . . . . . . . . . . . 3157

2. Linear Generative Grammars . . . . . . . . . . . . . 3182

3. Stepmatrices . . . . . . . . . . . . . . . . . . . 3203

4. From Phonons to the Speech Signal . . . . . . . . . . . 3233

5. Binary Tree Grammars . . . . . . . . . . . . . . . 3241

6. Conversion Grammars . . . . . . . . . . . . . . . 3256

7. Ensembles of Grammars . . . . . . . . . . . . . . . 3285

Hodge, Carleton $T$.

Afroasiatic: An Overview . . . . . . . . . . . . . . . . . . 6237

Hoenigswald, Henry M.

Introductory Remarks . . . . . . . . . . . . . . . . . . 1145

The Comparative Method . . . . . . . . . . . . . . . . . 1151

Hoijer, Harry

History of American Indian Linguistics . . . . . . . . . . . 10657

Hollyman, K. J.

French in the Pacific . . . . . . . . . . . . . . . . 8903

Horálek, $\mathrm{K}$.

Folk Poetry: History and Typology _. . . . . . . . . . . . 12741

Householder, Fred W. and Gregory Nagy

Greek

9735

Hymes, Dell

Anthropology and Sociology: An Overview . . . . . . . . . . . 121445 
XXXVII

Hymes, Dell and John Fought

American Structuralism . . . . . . . . . . . . . . . . 13903

Ikola, Osmo and Aulis J. Joki

Uralic . . . . . . . . . . . . . . . . . . . . . . . 91693

Ivanov, Vyacheslav V.

Growth of the Theoretical Framework of Modern Poetics . . . . . . 12835

Ives, Sumner, and Josephine Piekarz Ives

Linguistics and the Teaching of Reading and Spelling . . . . . . . . 10228

Jacob, Judith M.

Linguistics in Cambodja and on Cambodian . . . . . . . . . . . 2899

John, Vera P. and Marshall I. Peller

Language in Early Childhood Education . . . . . . . . . . . . 122101

Joki, Aulis J.

see Ikola, Osmo . . . . . . . . . . . . . . . . . . . . . . . . 91693

Kachru, Braj B.

Kashmiri and Other Dardic Languages . . . . . . . . . . . . . 5284

English in South Asia . . . . . . . . . . . . . . . . . . 5627

Kähler-Meyer, Emmi

Niger-Congo, Eastern Bantu . . . . . . . . . . . . . . . . 7307

Kaufman, Terrence

Areal Linguistics and Middle America . . . . . . . . . . . . . 11459

Kelkar, Ashok R.

General Linguistics in South Asia . . . . . . . . . . . 532

Kelley, Gerald

Telugu . . . . . . . . . . . . . . . . . . . . . 5382

Khan, Masud Husain

Urdu . . . . . . . . . . . . . . . . . . . . . . . . 5277

Khubchandani, Lachman $\mathbf{M}$.

Sindhi . . . . . . . . . . . . . . . . . . 5201

Killean, Carolyn G.

Classical Arabic . . . . . . . . . . . . . . . . . . . . 6413

Kiparsky, Paul

On Comparative Linguistics: The Case of Grassmann's Law . . . . . . 11115

Kiparsky, Valentin

Comparative and Historical Slavistics . . . . . . . . . . . . . . $\quad 194$

Koerner, E. F. K.

European Structuralism: Early Beginnings . . . . . . . . . 13717

Krauss, Michael E.

Eskimo-Aleut . . . . . . . . . . . . . . . . . . 10796

Na-Dene . . . . . . . . . . . . . . . . . . 10903

Krishnamurti, Bhadriraju

Comparative Dravidian Studies . . . . . . . . . . . . . 5309

Krueger, John R.

Altaic Languages: An Overview . . . . . . . . . . . . . . . 6179

Altaic Linguistic Reconstruction and Culture . . . . . . . . . 6569 


\section{XXXVIII}

Kuipers, Aert $\mathbf{H}$.

Caucasian . . . . . . . . . . . . . . . . . . . . . . 1315

Kurylowicz, Jerzy

Internal Reconstruction . . . . . . . . . . . . . . . . . 1163

Kutscher, Eduard Yechezkel

Aramaic. . . . . . . . . . . . . . . . . . . . . . . 6347

Labov, William

The Social Setting of Linguistic Change . . . . . . . . . . . . 11195

Lado, Robert

Applied Linguistics: Introduction . . . . . . . . . . . . . . 4375

Lafon, René

The Basque Language . . . . . . . . . . . . . . . . . . 91744

Landar, Herbert J.

The Tribes and Languages of North America: A Checklist . . . . . . 101253

Native North America . . . . . . . . . . . . . . . . . . 131331

Native Ibero-America . . . . . . . . . . . . . . . . . . 131359

Landau, Jacob M.

Language Study in Israel . . . . . . . . . . . . . . . . . 6721

Lastra, Yolanda

Literacy. . . . . . . . . . . . . . . . . . . . . . 4415

The Organization of Linguistic Activities . . . . . . . . . . . . 4607

Lavondès, Henry

see Wurm, Stephen A. . . . . . . . . . . . . . . . . . . 81025

Laycock, D. C.

English and Other Germanic Languages . . . . . . . . . . . . 8877

Laycock, D. C. , and C. L. Voorhoeve

History of Research in Papuan Languages . . . . . . . . . . . . 8509

Lazard, Gilbert

Persian and Tajik . . . . . . . . . . . . . . . . . . . . 664

Lea, Wayne A.

Computer Recognition of Speech . . . . . . . . . . . . . . 122765

Lehmann, W. P. , and Rolf Stachowitz

9688

Machine Translatic
Lepschy, Giulio C.

European Structuralism: Post-Saussurean Schools . . . . . . . . . 13887

Leslau, Wolf

Ethiopic and South Arabian . . . . . . . . . . . . . . . . 6467

Levin, Samuel R.

see Chatman, Seymour . . . . . . . . . . . . . . . . . . 10250

Lewis, E. Glyn

Linguistics and Second Language Pedagogy . . . . . . . . . . . 122131

Li, Fang-Kuei

Linguistics in Taiwan . . . . . . . . . . . . . . . . . . 2177

Lieberman, Philip

A Study of Prosodic Features . . . . . . . . . . . . . . . 122419 
Lisker, Leigh

On Time and Timing in Speech . . . . . . . . . . . . . . . 122387

Longacre, Robert E.

Comparative Reconstruction of Indigenous Languages . . . . . . . 4320

Introductory Remarks . . . . . . . . . . . . . . . . . . 11287

Lotz, John $\dagger$

Metrics . . . . . . . . . . . . . . . . . . . 12963

Lukoff, Fred

Linguistics in the Republic of Korea . . . . . . . . . . . . . 2735

Lunt, Horace

Yugoslavia . . . . . . . . . . . . . . . . . . . . . . 1562

Luria, A. R.

Basic Problems of Neurolinguistics . . . . . . . . . . . . . . 122561

McClaran, Marlys

Mexico . . . . . . . . . . . . . . . . . . . 101079

McDavid, Jr. , Raven I.

The English Language in the United States . . . . . . . . . . . 105

McDonald, Eugene T.

Speech Pathology . . . . . . . . . . . . . . . . . . . 122641

McKaughan, Howard

Minor Languages of the Philippines . . . . . . . . . . . . . . 8155

MacKenzie, D. N.

Iranian Languages . . . . . . . . . . . . . . . . . . . 5450

McQuown, Norman A.

Linguistics of Non-Ibero-American Languages: Introduction . . . . . 4251

Malécot, André

Cross-Language Phonetics . . . . . . . . . . . . . . . . . 122507

Malino, Jonathan

see Bar-Hillel, Yehoshua . . . . . . . . . . . . . . . . . 1237

Malkiel, Yakov

Genetic Analysis of Word Formation . . . . . . . . . . . . . 3305

Prefatory Note . . . . . . . . . . . . . . . . . . 3305

I. The Place of Affixation in the Edifice of Language . . . . . 3307

II. The Genesis of a Derivational Suffix . . . . . . . . . . 3323

III. Diffusion of Derivational Suffixes . . . . . . . . . . . 3333

IV. The Esthetic Dimension of Derivation . . . . . . . . . . 3346

V. Prospects of Future Research . . . . . . . . . . . . . 3355

Hispanic Philology . . . . . . . . . . . . . . . . . . . 4158

General Diachronic Linguistics . . . . . . . . . . . . . . . 982

Comparative Romance Linguistics . . . . . . . . . . . . . . 9835

Malmberg, Bertil

Descriptive Linguistics . . . . . . . . . . . . . . . . . . 9223

Marckwardt, Albert H.

General Educational Aims of Native Language Teaching and Learning . . 10206 
$\mathrm{XL}$

Marcus, Solomon

Mathematical Linguistics in Europe . . . . . . . . . . . . . . 9646

Linguistics as a Pilot Science . . . . . . . . . . . . . . . . 122871

Margalit, Avishai

see Bar-Hillel, Yehoshua . . . . . . . . . . . . . . . . . 1237

Markey, Thomas L. see Haugen, Einar c . . . . . . . . . . . . . . . . . . . . . . 91461

Martínez, Fernando Antonio

General and Ibero-American Linguistics: Lexicography . . . . . . . 484

Masica, Colin

see Ramanujan, A. K. . . . . . . . . . . . . . . . . . . . 5543

Mattingly, Ignatius G.

Speech Synthesis for Phonetic and Phonological Models . . . . . . . 122451

Mayers, Marvin K.

Indigenous Dialectology . . . . . . . . . . . . . . . . . 4310

Meid, Wolfgang

Old Celtic Languages . . . . . . . . . . . . . . . . . . 91190

Miller, Roy Andrew

The Tibeto-Burman Languages of South Asia . . . . . . . . . . 5431

The Far East . . . . . . . . . . . . . . . . . . . . . 131213

Milner, George B.

Fijian and Rotuman . . . . . . . . . . . . . . . . . . . 8397

Miltner, Vladimír

Hindi . . . . . . . . . . . . . . . . . . . . . . 555

Moravcsik, J. M. E.

Linguistics and Philosophy . . . . . . . . . . . . . . . . 123

Morpurgo-Davies, A.

Language Classification in the Nineteenth Century . . . . . . . . . 13607

Moulton, William G.

Geographical Linguistics . . . . . . . . . . . . . . . . . 9196

Nagy, Gregory

see Householder, Fred W. . . . . . . . . . . . . . . . . . 9735

Nida, Eugene A.

Translation . . . . . . . . . . . . . . . . . 121045

Nketia, J. H. Kwabena

Surrogate Languages of Africa . . . . . . . . . . . . . . . 7699

The Linguistic Aspect of Style in African Languages . . . . . . . . 7733

Oftedal, Magne

Modern Celtic Languages . . . . . . . . . . . . . . . . . 91202

O'Grady, Geoffrey N.

Lexicographic Research in Aboriginal Australia . . . . . . . . . . 8779

O'Grady, Geoffrey N. , and Charles A. Zisa

Checklist of Oceanic Language and Dialect Names . . . . . . . . . 81189

Oksaar, Els

Bilingualism . . . . . . . . . . . . . . . . . . . . 9476 
Ornstein, Jacob

Foreign Language Teaching . . . . . . . . . . . . . . . . 1143

Palmer, F. R.

Cushitic . . . . . . . . . . . . . . . . . . . . . 6571

Pandit, Prabodh B.

Gujarati . . . . . . . . . . . . . . . . . . . 5105

Paper, Herbert $\mathrm{H}$.

Indo-European Languages: An Overview . . . . . . . . . . . . 63

Pattanayak, D. P.

Oriya and Assamese . . . . . . . . . . . . . . . . . . . 5122

Pei, Mario A.

Artificial Languages: International (Auxiliary) . . . . . . . . . . . 12999

Peller, Marshall I. , and Vera P. John

Language in Early Childhood Education . . . . . . . . . . . . 122101

Penzl, Herbert

Old Germanic Languages . . . . . . . . . . . . . . . 91232

Percival, W. Keith

The Grammatical Tradition and the Rise of the Vernaculars . . . . . 13231

Piekarz Ives, Josephine

see Ives, Sumner . . . . . . . . . . . . . . . . . . . . 10228

Pike, Kenneth L.

A Guide to Publications Related to Tagmemic Theory . . . . . . . 3365

1. Relativity of Description and Theory to the Observer . . . . . . 3366

2. Complementarity of Perspective . . . . . . . . . . . . 3367

3. Field as Dimensional Matrix . . . . . . . . . . . . . 3367

4. Wave as Nucleus plus Margin . . . . . . . . . . . . . . 3376

A. In Phonology . . . . . . . . . . . . . . . . . . 3377

B. In Grammar . . . . . . . . . . . . . . . . . 3379

C. In Lexicon and Culture . . . . . . . . . . . . . . . 3380

5. Particle as Structured Unit . . . . . . . . . . . . 3380

A. In Grammar Units . . . . . . . . . . . . . . . 3381

B. In Phonology . . . . . . . . . . . . . . . . . . 3389

C. In Lexicon and Culture . . . . . . . . . . . . . . 3390

Index . . . . . . . . . . . . . . . . . . . . . 3391

Appendix . . . . . . . . . . . . . . . . . . . . 3393

Pinborg, Jan

Classical Antiquity: Greece . . . . . . . . . . . . . . . . 1369

Polotsky, H. J.

Coptic . . . . . . . . . . . . . . . . . . . . . . . . . . . . 6558

Poppe, Nicholas

Altaic . . . . . . . . . . . . . . . . . . . . 1301

The Mongolian Peoples Republic . . . . . . . . . . . . . . 2758

Pride, J. B.

Sociolinguistics . . . . . . . . . . . . . . . . . . . . 121607

Quemada, Bernard

Lexicology and Lexicography . . . . . . . . . . . . . . . 9395 
XLII

Rabin, Chaim

Hebrew . . . . . . . . . . . . . . . . . . . . . . . 6304

Ramanujan, A. K. , and Colin Masica

Toward a Phonological Typology of the Indian Linguistic Area . . . . 5543

Rea, John A.

The Romance Data of the Pilot Studies for Glottochronology . . . . . 11355

Read, Allen Walker

Approaches to Lexicography and Semantics . . . . . . . . . . . 10145

Redard, Georges

Other Iranian Languages

Reid, Lawrence Andrew

Diachronic Typology of Philippine Vowel Systems . . . . . . . . 11485

Reiner, Erica

Akkadian . . . . . . . . . . . . . . . . . . . . . . 6274

Renou, Louis $\dagger$

Panini . . . . . . . . . . . . . . . . . . . 5481

Rensch, Calvin R.

Otomanguean Isoglosses . . . . . . . . . . . . . . . . . . . . 11295

Roberts, A. Hood and Michael Zarechnak

Mechanical Translation . . . . . . . . . . . . . . 122825

Robins, R. H.

The History of Language Classification . . . . . . . . . . . . 113

Rocher, Rosane India . . . . . . . . . . . . . . . . . . . . . . . . 133

Romeo, Luigi

Classical Antiquity: Rome . . . . . . . . . . . . . . . . . 13127

Rossi-Landi, Ferruccio

Linguistics and Economics . . . . . . . . . . . . . . . . 121787

Roth, George

see Werner, Oswald . . . . . . . . . . . . . . . . . . . . . . . . . 121477

Rubenstein, Herbert

Psycholinguistics: An Overview . . . . . . . . . . . . . 121071

Computer Applications: An Overview . . . . . . . . . . . . . 12271

Rudnyćkyj, J. B.

Immigrant Languages, Language Contact, and Bilingualism in Canada . . 10592

Samarin, William $\mathbf{J}$.

Adamawa-Eastern . . . . . . . . . . . . . . . . . . . 7213

Sankoff, David

Mathematical Developments in Lexicostatistic Theory . . . . . . . 1193

Sapir, J. David

West Atlantic: An Inventory of the Languages, their Noun Class Systems, and Consonant Altemation . . . . . . . . . . . . . . . . 745

Saporta, Sol

General and Ibero-American Linguistics: Introduction . . . . . . . 43

Sarles, Harvey B.

Facial Expression and Body Movement . . . . . . . . . . 12297 
Sathasivam, A.

Linguistics in Ceylon, (II): Tamil . . . . . . . . . . . . . . 5752

Sawashima, Masayuki

Laryngeal Research in Experimental Phonetics . . . . . . . . . . 122303

Schachter, Paul

The Present State of African Linguistics . . . . . . . . . . . . 730

Schepers, Emile

see Werner, Oswald

$12 \quad 1477$

Schmalstieg, William R.

Lithuanian . . . . . . . . . . . . . . . . . . . . . . 1287

Schmidt, Siegfried J.

see Hartmann, Peter . . . . . . . . . . . . . . . . . . . 93

Schmitter, Peter

see Gipper, Helmut . . . . . . . . . . . . . . . . . . . 13481

Schooneveld, Cornelis H. van

Morphemics . . . . . . . . . . . . . . . . . . 122

Schramm, Gene M.

The Semitic Languages: An Overview . . . . . . . . . . . . 6257

Sebeok, Thomas A.

Semiotics: A Survey of the State of the Art . . . . . . . . . . . 12211

Editor's Introduction . . . . . . . . . . . . . . . . . . $1 \mathrm{v}$

Editor's Introduction . . . . . . . . . . . . . . . . . . 2 v

Editor's Introduction . . . . . . . . . . . . . . . . . $3 \mathrm{v}$

Editor's Introduction . . . . . . . . . . . . . . . . . . 4 vii

Editor's Introduction . . . . . . . . . . . . . . . . . . 5 vii

Editor's Introduction . . . . . . . . . . . . . . . . . . . 6 vii

Editor's Introduction . . . . . . . . . . . . . . . . . . . . . 7 v

Editor's Introduction . . . . . . . . . . . . . . . . . . $8 \mathrm{v}$

Editor's Introduction . . . . . . . . . . . . . . . . . . $9 \mathrm{v}$

Editor's Introduction . . . . . . . . . . . . . . . . . . . $10 \mathrm{v}$

Editor's Introduction . . . . . . . . . . . . . . . . . . . $11 \mathrm{v}$

Editor's Introduction . . . . . . . . . . . . . . . . . . $12 \mathrm{v}$

Editor's Introduction . . . . . . . . . . . . . . . . . . $13 \mathrm{v}$

Shands, Harley C.

Language and Psychiatry . . . . . . . . . . . . . . . 122657

Shetter, William Z.

Netherlandic and Frisian . . . . . . . . . . . . . . . . . 91383

Sherzer, Joel

Areal Linguistics in North America . . . . . . . . . . . . . . 10749

Shevelov, George Y.

Belorussian and Ukrainian . . . . . . . . . . . . . . . . . 1217

Shipley, William F.

California . . . . . . . . . . . . . . . . . . 101046

Sibayan, Bonifacio

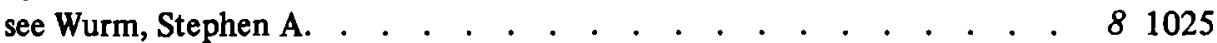


Slama-Cazacu, Tatiana

The Study of Child Language in Europe . . . . . . . . . . . . 9512

Smith, W. John

Zoosemiotics: Ethology and the Theory of Signs . . . . . . . . . 12561

Sorsby, William

Maps: Tribal Groups of North America and Central America . . . . . 101442

Southworth, Franklin C.

Marathi . . . . . . . . . . . . . . . . . . . . 599

Spencer, John

Colonial Language Policies and their Legacies . . . . . . . . 7537

Spolsky, Bernard

Linguistics and Education: An Overview . . . . . . . . . . . . 122021

Linguistics and the Language Barrier to Education . . . . . . . . . 122027

Staal, J. F.

Sanskrit Philosophy of Language . . . . . . . . . . . . . . 5499

Stachowitz, Rolf

see Lehmann, W. P. . . . . . . . . . . . . . . . . . . . 9688

Stankiewicz, Edward

Poland . . . . . . . . . . . . . . . . . . . . . 1538

Appendix II: Slavic Morphophonemics in its Typological and Diachronic

Aspects . . . . . . . . . . . . . . . . . . . . . . 3495

Structural Poetics and Linguistics . . . . . . . . . . . . . . 12629

Bibliography of the History of Linguistics . . . . . . . . . . . 131381

Steger, Hugo

Middle High and New High German . . . . . . . . . . . . . 91282

Stewart, John M.

Niger-Congo, Kwa . . . . . . . . . . . . . . . . . . . 7179

Stipa, Gunter J.

Uralic

Stokoe, Jr. , William C.

Classification and Description of Sign Languages . . . . . . . . . 12345

Strevens, Peter

Language Teaching . . . . . . . . . . . . . . . . . . . 9702

Studdert-Kennedy, Michael

The Perception of Speech . . . . . . . . . . . . . . . . . 122349

Suárez, Jorge A.

Linguistics of Non-Ibero-American Languages: Classical Languages . . . 4254

Subramoniam, Vadasery I.

Malayalam . . . . . . . . . . . . . . . . . . . . . 5372

Sugimoto, $T$.

Japanese in Taiwan . . . . . . . . . . . . . . . . . . . . . 8969

Suzuko, Tamura

Studies of the Ainu Language . . . . . . . . . . . . . . . 2608

Svejkovský, F.

Theoretical Poetics in the Twentieth Century . . . . . . . . . . 12863 
Szemerényi, Oswald

Comparative Linguistics . . . . . . . . . . . . . . . . . 9119

Taranovski, Kiril

Metrics . . . . . . . . . . . . . . . . . . . . . . . 1192

Taylor, Orlando L. , and Joseph P. Fox

Language Behavior and Disorders Associated with Brain Damage . . . . 122595

Teeter, Karl V.

Algonquian .'. . . . . . . . . . . . . . . . . . . . 10.1143

Terry, Robert R.

Chadic . . . . . . . . . . . . . . . . . . . . . 7443

Thomas, David D.

see Thompson, Laurence C. . . . . . . . . . . . . . . . . 2815

Thompson, Laurence C. , and David D. Thomas

Vietnam .. . . . . . . . . . . . . . . . . 2815

Thompson, Laurence $\mathbf{C}$.

The Northwest . . . . . . . . . . . . . . . . . . . . 10979

Todorov, Tzvetan

Literary Genres . . . . . . . . . . . . . . . . . . . . 12957

Toporov, V.N.

Folk Poetry: General Problems . . . . . . . . . . . . . . . 12683

Torres Quintero, Rafael

see Guitarte, Guillermo L. . . . . . . . . . . . . . . . . . 4562

Toru, Mineya

Languages of South East Asia . . . . . . . . . . . . . . . 2683

Toshio, Yamada

The Writing System: Historical Research and Modern Development . . . 2693

Trager, George L.

Writing and Writing Systems . . . . . . . . . . . . . . . . 12373

Trifonovitch, Gregory J.

see Wurm, Stephen A. . . . . . . . . . . . . . . . . . . 81025

Troike, Rudolph C.

Linguistics and the Language Arts in Elementary and Secondary Education 122117

Tucker, A. N.

Orthographic Systems and Conventions in Sub-Saharan Africa . . . . . 7618

Uhlenbeck, E. M.

Indonesia and Malaysia . . . . . . . . . . . . . . . . . . 2847

Indonesia and Malaysia . . . . . . . . . . . . . . . . 855

Ullendorff, Edward

Comparative Semitics . . . . . . . . . . . . . . . . . . 6261

Ullmann, Stephen

Semantics . . . . . . . . . . . . . . . . . . . . . . 9343

Umiker, Donna Jean

Speech Surrogates: Drum and Whistle Systems . . . . . . . . . . 12497

Uriarte, Luis

see Werner, Oswald . . . . . . . . . . . . . . . . . . . 121477 
XLVI

Vachek, Josef

Middle and Modern English . . . . . . . . . . . . . 91407

Valdman, Albert

Some Aspects of Decreolization in Creole French . . . . . . . . . 11507

Valkhoff, Marius F.

Descriptive Bibliography of the Linguistics of Afrikaans: A Survey of

Major Works and Authors . . . . . . . . . . . . . . . 7455

Venezky, Richard L.

Theoretical and Experimental Bases for Teaching Reading . . . . . . 122057

Vergote, J.

Egyptian . . . . . . . . . . . . . . . . . . . . . . 6531

Vinay, Jean-Paul

Le français en Amérique du Nord: Problèmes et réalisations . . . . . 10323

Bibliographie . . . . . . . . . . . . . . . . . . . . . 10414

Voegelin, C. F. and F. M.

The Southwest and Great Basin . . . . . . . . . . . . . 101100

Voorhoeve, C. L.

see Laycock, D. C. . . . . . . . . . . . . . . . . . . . 8509

Waldman, Nahum M.

The Hebrew Tradition . . . . . . . . . . . . . . . . . 131285

Wang, William S-Y.

Bibliography of Chinese Linguistics . . . . . . . . . . . . . . 2188

Approaches to Phonology . . . . . . . . . . . . . . . . . 10101

Watson, O. Michael

Proxemics . . . . . . . . . . . . . . . . . . . . . . 12311

Weinreich, Uriel

Lexicology . . . . . . . . . . . . . . . . . . . . . . 160

Explorations in Semantic Theory . . . . . . . . . . . . . 3395

1. Introduction . . . . . . . . . . . . . . . . . . . 3395

2. The Semantic Theory KF: A Critical Analysis . . . . . . . . 3397

3. A New Semantic Theory . . . . . . . . . . . . . . . 3417

4. Concluding Remarks . . . . . . . . . . . . . . . . 3467

Bibliography . . . . . . . . . . . . . . . . . . . . 3474

Welmers, Wm. E

Niger-Congo, Mande . . . . . . . . . . . . . . . . . . 7113

Christian Missions and Language Policies . . . . . . . . . . . . 7559

Checklist of African Language and Dialect Names . . . . . . . . . 7759

Werner, Oswald, William Hagedorn, George Roth, Emile Schepers and

Luis Ariarte

Some New Developments in Ethnosemantics and the Theory and Practice

of Lexical/Semantic Fields

Westphal, E. O. J.

The Clici Languages of Southern and Eastern Africa . . . . . . . . 7367

Whiteley, W. H.

Language Policies of Independent African States . . . . . . . . . 7548 
Wigdorsky, Leopoldo

see Gomes de Matos, Francisco . . . . . . . . . . . . . . . . . 4464

Williamson, Kay

The Benue-Congo Languages and lijo . . . . . . . . . . . . . 7245

Winter, Werner

Indo-European . . . . . . . . . . . . . . . . . . . . 1205

Areal Linguistics: Some General Considerations . . . . . . . . . 11135

Worth, Dean

Syntax . . . . . . . . . . . . . . . . . . . . . . . 135

Paleosiberian . . . . . . . . . . . . . . . . . . . . . 1345

Wurm, Stephen A.

The Papuan Linguistic Situation . . . . . . . . . . . . . . . 8541

Classifications of Australian Languages, including Tasmanian . . . . . 8721

Pidgins, Creoles and Lingue Franche . . . . . . . . . . . . . 8999

Language Policy, Language Engineering, and Literacy (New Guinea and

Australia), Bonifacio Sibayan (the Philippines), Gregory J. Trifonovitch

(Trust Territory of the Pacific Islands), S. T. Alisjahbana (Indonesia and

Malaysia), and Henri Lavondes (French Polynesia) . . . . . . . . . 81025

Wylie, Turrell V.

Tibet. . . . . . . . . . . . . . . . . . . . 2766

Yalman, Nur

The Semantics of Kinship in South India and Ceylon . . . . . . . . 5607

Yar-Shater, Ehsan

Iran and Afghanistan . . . . . . . . . . . . . . . . . . 6669

Zarechnak, Michael, and A. Hood Roberts

Mechanical Translation . . . . . . . . . . . . . . . . . . 122825

Zeps, Valdis J.

Latvian . . . . . . . . . . . . . . . . . . . . 1265

Zide, Norman $\mathrm{H}$.

Munda and Non-Munda Austroasiatic Languages . . . . . . . . . 5411

Zisa, Charles A.

see O'Grady, Geoffrey N. . . . . . . . . . . . . . . . . . 81189

Zvelebil, Kamil V.

Tamil 
